\title{
Desmoplastic variant of ameloblastoma of the maxilla: A case report
}

\author{
Kwang-Joon Koh ${ }^{1, *}$, Ha-Na Park ${ }^{1}$, Kyoung-A Kim ${ }^{1}$ \\ ${ }^{1}$ Department of Oral and Maxillofacial Radiology, School of Dentistry and Institute of Oral Bioscience, Chonbuk National University, \\ Jeonju, Korea
}

\section{ABSTRACT}

The desmoplastic variant of ameloblastoma is a rare form of ameloblastoma characterized by unique radiographic and histologic features. A 46-year-old female was referred to our hospital, complaining of swelling in the left upper lip area. Radiographic findings revealed an ill-defined multilocular lesion with a large cystic lesion and thick sclerotic trabeculae on the left anterior maxilla. After the patient underwent partial osteotomy, histologic analysis revealed a desmoplastic ameloblastoma with no evidence of a hybrid lesion or cyst formation. The radiographic findings in the present case were different from those described in previous case reports. These findings are of special importance due to the unfamiliar radiographic and histologic features of this lesion.(Imaging Sci Dent 2015; 45: 241-5)

KEY WORDS: Ameloblastoma; Jaw; Neoplasms

Desmoplastic ameloblastoma is a rare variant of ameloblastoma characterized by unique radiographic and histologic features. ${ }^{1-3}$ This tumor shows a predilection for the anterior maxilla ${ }^{3-5}$ and occurs most commonly in the third through seventh decades of life. ${ }^{6-8}$ It accounts for $4 \%-5 \%$ of all ameloblastomas, ${ }^{6,8}$ and usually presents as a painless, expansile mass. ${ }^{3}$ The radiographic features of desmoplastic ameloblastoma differ markedly from those of other ameloblastoma variants. Radiographically, it either exhibits a mixed radiolucent/radiopaque lesion or presents with multilocular radiolucencies that have ill-defined or well-defined borders. ${ }^{2}$ It usually shows cortical expansion and tooth displacement, but it rarely shows root resorption. Histologically, it shows abundant, densely collagenous stroma containing small nests and strands of odontogenic epithelium. ${ }^{9}$ This report describes a rare case of desmoplastic ameloblastoma characterized by peculiar radiographic features.

Received June 22, 2015; Revised July 23, 2015; Accepted August 2, 2015

*Correspondence to : Prof. Kwang-Joon Koh

Department of Oral and Maxillofacial Radiology, School of Dentistry, 20 Geonjiro, Deokjin-gu, Jeonju-si, Jeollabuk-do 54907, Korea

Tel) 82-63-250-2023, Fax) 82-63-250-2081, E-mail) kkj1512@jbnu.ac.kr

\section{Case Report}

A 46-year-old female visited Chonbuk National University Dental Hospital with the complaint of swelling in the left upper lip area. She had begun to notice the painless swelling two months previously. Her past medical history was non-remarkable. A clinical examination found a bony and hard gingival swelling that extended from the left maxillary incisors to the premolar regions, as well as displacement of the left maxillary lateral incisor and canine. Neither tenderness nor pus discharge was observed upon palpation. A panoramic radiograph (Fig. 1) and a periapical radiograph (Fig. 2) showed an ill-defined multilocular radiolucency with a large cystic lesion extending from the left upper central incisor to the premolar area. Thick sclerotic trabeculae were observed in the lesion, as well as displacement of the left upper lateral incisor and canine. However, no loss of lamina dura or root resorption was observed. Facial computed tomographic (CT) images (Fig. 3) revealed an ill-defined multilocular lesion with a cystic lesion and thick trabeculae on the left anterior maxilla. Thinning, expansion, and perforation of the buccal and palatal cortical plates were noted. The lesion expanded into the left maxillary sinus with destruction of the anteromedial wall and mucosal thickening. With the tentative

Copyright (c) 2015 by Korean Academy of Oral and Maxillofacial Radiology

This is an Open Access article distributed under the terms of the Creative Commons Attribution Non-Commercial License (http://creativecommons.org/licenses/by-nc/3.0) which permits unrestricted non-commercial use, distribution, and reproduction in any medium, provided the original work is properly cited. Imaging Science in Dentistry · pISSN 2233-7822 eISSN 2233-7830 

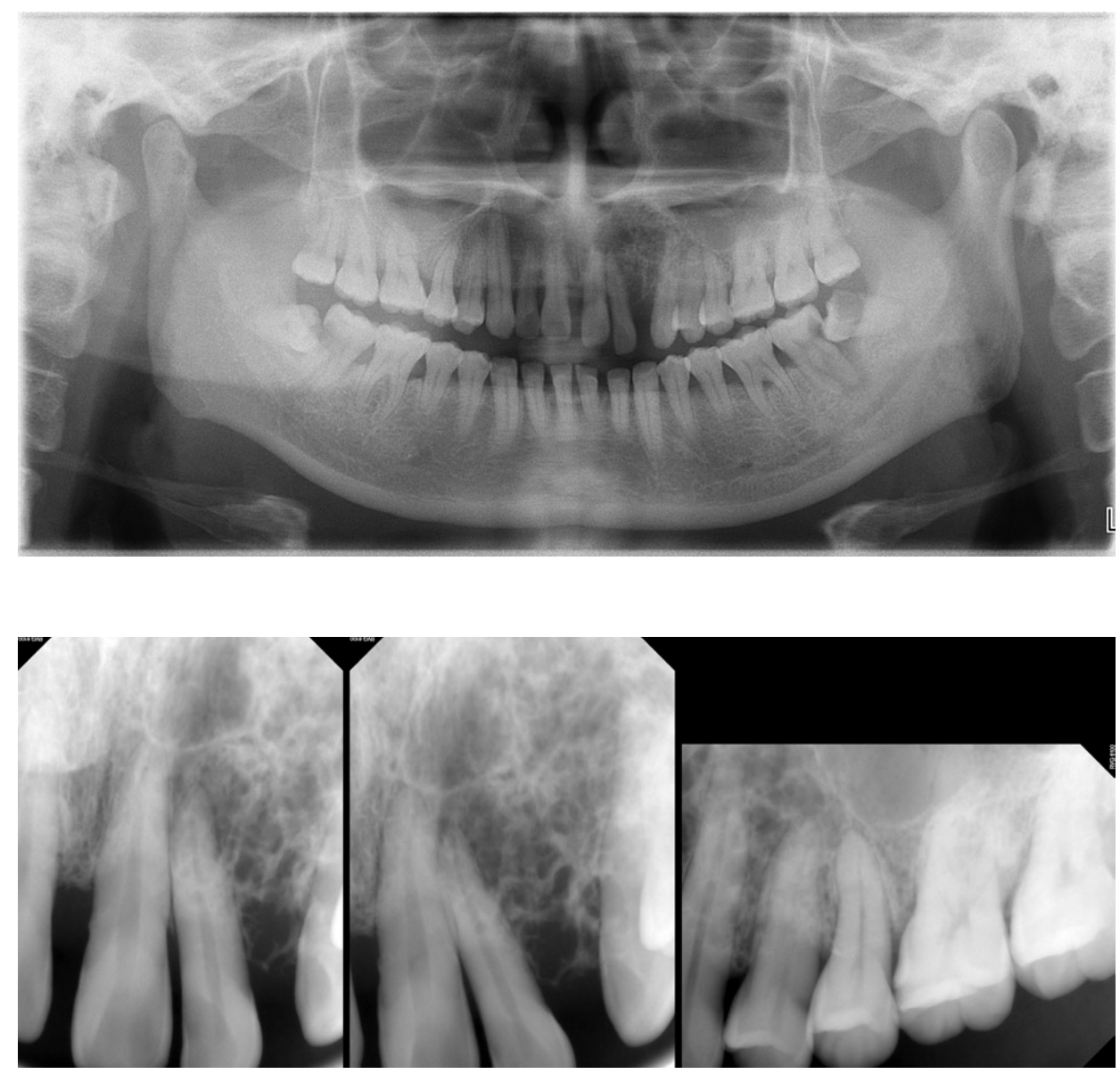

Fig. 1. A panoramic radiograph shows an ill-defined multilocular radiolucency with a large cystic lesion extending from the left upper central incisor to the premolar region and displacement of the left upper lateral incisor and canine.
Fig. 2. Periapical radiographs show a large cystic lesion on the apical region of the left upper central incisor area and an ill-defined multilocular radiolucency with thick sclerotic trabeculae on the left upper anterior maxilla with displacement of the adjacent lateral incisor and canine. diagnosis of an odontogenic myxoma or ameloblastoma, the patient underwent partial osteotomy. After an operation, a biopsy was performed, and the histologic analysis (Fig. 4) revealed a non-encapsulated mass with small, scattered tumor nests of epithelium in the fibrous stroma. Small areas of tumor nests showed some columnar cells with reversed nuclear polarity. The stroma showed extensive desmoplasia and new bone formation with osteoblastic rimming. The specimen was consistent with desmoplastic ameloblastoma. The patient has been disease-free for three years since the operation and is under routine follow-up care. The present case differed from the lesions described in previous case reports in that it presented with a multilocular radiolucency that had thick sclerotic trabeculae, and showed no root resorption of the involved teeth.

\section{Discussion}

Ameloblastoma is a benign but locally aggressive polymorphic neoplasm that consists of proliferating odontogenic epithelium. ${ }^{7}$ Three types of intraosseous ameloblas- tomas exist: the conventional or solid/multicystic variant, the unicystic variant, and the desmoplastic variant. ${ }^{10}$

In 1984, Eversole et al. ${ }^{11}$ first described the desmoplastic variant of ameloblastoma, which is distinguished from conventional ameloblastoma based on its tendency to involve the anterior maxilla ${ }^{5}$ and its unique appearance. ${ }^{2}$ Desmoplastic ameloblastoma also differs from other types of ameloblastoma in that it is located in the anterior or premolar regions of the maxilla or mandible, and its radiographic appearance is often more typical of a mixed lesion. Some authors have suggested that desmoplastic ameloblastoma tends to exhibit rapid growth ${ }^{3-5}$ and progressive behavior. ${ }^{8}$ Desmoplastic ameloblastoma accounts for $4 \%$ $5 \%$ of all ameloblastomas. ${ }^{6,8}$ It occurs in patients in the third through seventh decades of life. ${ }^{6-8}$ A clinicopathological analysis of the 68 cases of desmoplastic ameloblastoma that have previously been reported found that more than $70 \%$ of the cases occurred in the anterior and premolar regions of the jaw. ${ }^{7}$ Contrary to reports arguing that desmoplastic ameloblastoma has an anterior maxillary predilection, Effiom and Odukoya ${ }^{12}$ reported that $81 \%$ of cases showed a mandibular predilection, with $82 \%$ of cas- 

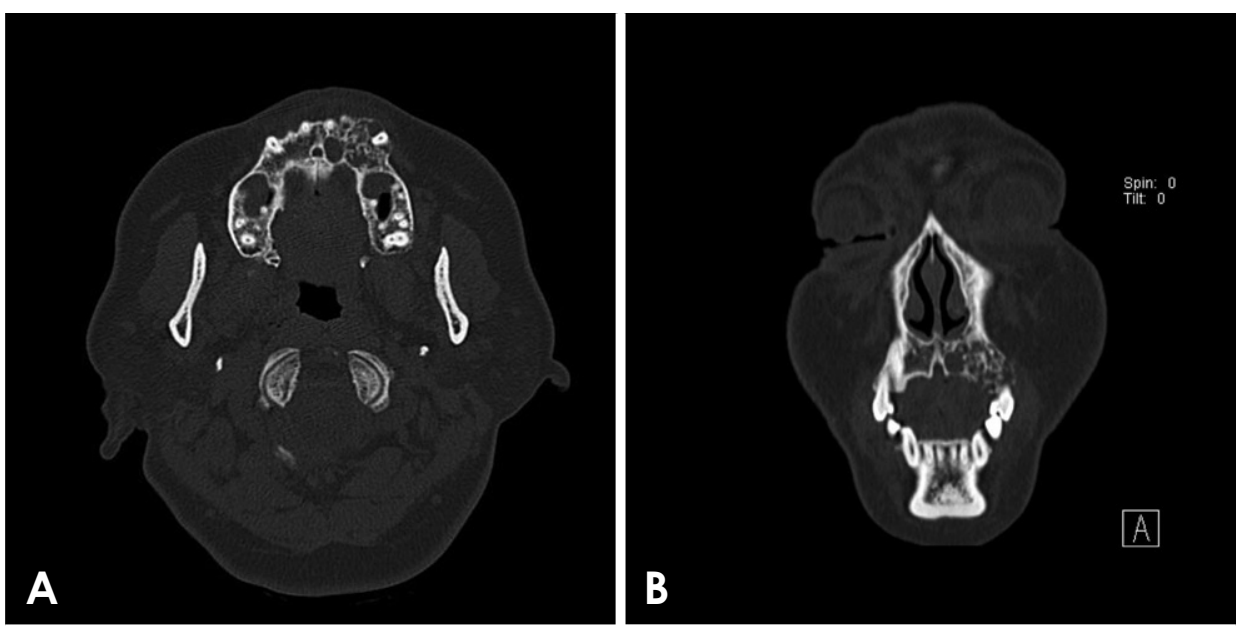

Fig. 3. The axial (A), coronal (B) computed tomographic (CT) images show an ill-defined multilocular lesion with a large cystic lesion, thick trabeculae, expansion, and perforation of the both buccal and palatal cortical plates. C. A sagittal CT image shows an ill-defined multilocular radiolucency with a large cystic lesion. D. A sagittal CT image shows bony expansion to the left maxillary sinus.
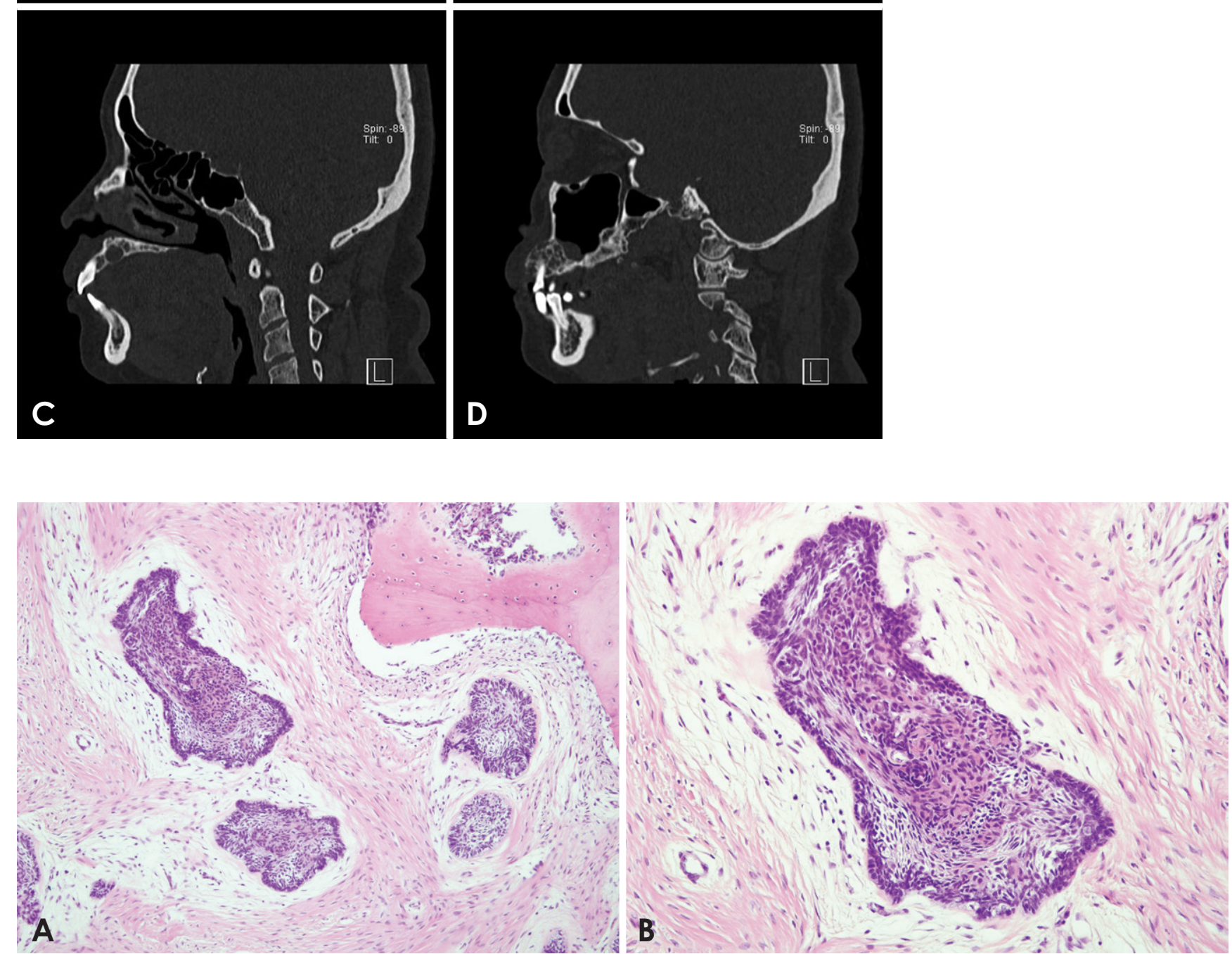

Fig. 4. Photomicrographs show small, scattered tumor nests and new bone formation, as well as desmoplastic changes in the stroma. A. H\&E stain, $100 \times$. B. H\&E stain, $200 \times$.

es showing radiolucency.

Conventional ameloblastoma predominantly occurs in patients in the third through fifth decades of life, compris- ing $92 \%$ of cases in this age group. ${ }^{13}$ Unilocular forms have been found to be significantly more common in younger patients. ${ }^{14}$ It has been well established that the majority of 
multilocular ameloblastomas arise in the mandible, with most in the molar and ramus regions. The present case showed multilocular radiolucency with a large cystic lesion of the anterior maxilla.

Desmoplastic ameloblastoma appears as a diffuse, mixed radiolucent and radiopaque lesion with a honeycomb or soap-bubble pattern. ${ }^{2}$ Kawai et al. ${ }^{2}$ reported that $25 \%$ of cases showed a mixed radiolucent and radiopaque pattern with new bone formation. The remainder of the cases were radiolucent.

MacDonald-Jankowski et al. ${ }^{14}$ found a significantly higher proportion of unilocular lesions with well-defined margins in conventional ameloblastomas, but Poon et al. ${ }^{15}$ reported that only $16 \%$ of cases showed unilocular lesions.

However, Kaffe et al. ${ }^{7}$ reported that desmoplastic ameloblastoma appeared as a multilocular lesion in $20 \%$ of cases and as a unilocular lesion in $33 \%$ of cases, while the remaining $47 \%$ of lesions were not loculated. Unlike other ameloblastomas, desmoplastic ameloblastoma has poorly defined borders, ${ }^{7,16}$ and it may be mistaken for a benign fibro-osseous lesion. ${ }^{6,7}$ A CT scan is used to obtain a three-dimensional image of the lesion and to better examine the soft tissues. ${ }^{10}$ Although ameloblastoma is more likely than odontogenic myxoma to appear as a unilocular lesion, ${ }^{14}$ these two tumors have no significant differences regarding locularity.

Lo Muzio et al. ${ }^{17}$ reported that six of 10 odontogenic myxomas were multilocular and four were unilocular. Therefore, other radiographic features are required to differentiate ameloblastoma and odontogenic myxoma. One such feature is the border between the lesion and the adjacent normal tissue. MacDonald-Jankowski et al. ${ }^{14}$ reported that ameloblastoma was significantly better defined than odontogenic myxoma. The unique radiographic features of the desmoplastic variant of ameloblastoma, which often give the impression of a fibro-osseous lesion, seem to reflect the characteristic aggressive behavior of the tumor in many cases. This may also explain the ill-defined border of desmoplastic ameloblastoma. ${ }^{7,18}$

Kaffe et al. ${ }^{7}$ and Li et al ${ }^{16}$ reported tooth displacement in $48 \%-92 \%$ of desmoplastic ameloblastomas, whereas root resorption was found in 8.7\%-33\% of desmoplastic ameloblastomas. However, Lo Muzio et al. ${ }^{17}$ reported root resorption in $20 \%$ of odontogenic myxomas.

One advantage of $\mathrm{CT}$ over magnetic resonance imaging is the ability to distinguish desmoplastic ameloblastoma from other fibro-osseous lesions through the detection of thick bony trabeculae. ${ }^{19}$ In several reports, tumors were incorrectly diagnosed as fibro-osseous lesions based on the history, clinical findings, and radiographic appearance. ${ }^{7}$

The present case showed an ill-defined expansile multilocular radiolucency with perforation of the cortical plate, unlike the encapsulated mixed radiolucent and radiopaque pattern previously described for these lesions. Furthermore, the present case showed a thick trabecular pattern with tooth displacement. Therefore, odontogenic myxoma and ameloblastoma were included in the differential diagnosis.

A biopsy was performed for the final diagnosis of the lesion. Desmoplastic ameloblastoma appears as compressed islands and thin cords of ameloblastic epithelial cells embedded in a dense collagenized stroma. ${ }^{7}$ Areas of stellate reticulum are rare to absent. ${ }^{10}$ The present case involved a non-encapsulated mass with small, scattered tumor nests of epithelium in the fibrous stroma. An invasive growth pattern of this type into marrow spaces and the lack of demarcation with fibrous connective tissues are correlated with the indistinct border observed in desmoplastic ameloblastoma., ${ }^{2,21}$ The non-encapsulated nature of the mass seems to be related to the ill-defined border, and is an important difference from well-defined fibro-osseous lesions.

Histologically, cystic changes in the tumor mass of desmoplastic ameloblastoma have been reported. ${ }^{6,19,20}$ However, no cystic changes were found in the large cystic lesion of the present case. A desmoplastic ameloblastoma with features of other histologic types is considered to be a hybrid lesion. ${ }^{5}$ A hybrid ameloblastoma is composed of desmoplastic ameloblastoma and conventional follicular or plexiform ameloblastoma. ${ }^{21,22}$ The present case showed no histological findings characteristic of conventional follicular or plexiform ameloblastoma. Therefore, the lesion in our case was not hybrid ameloblastoma.

Desmoplastic ameloblastoma often shows prominent bone formation. ${ }^{6,19}$ Thompson et al. ${ }^{19}$ suggested that the production of new bone results from an attempt to repair the damaged laminated bone trabeculae resorbed by the tumor expansion. Kawai et al. ${ }^{2}$ proposed that the adjacent bone trabeculae tend to persist because osteoblastic activity is more vigorous than osteoclastic activity and may be induced by newly formed bony trabeculae. The present case showed prominent new bone formation with osteoblastic rimming in the solid mass. The new bone formation may have been reflected by the thick sclerotic trabeculae seen on the radiographs.

Most cases of desmoplastic ameloblastoma have been described as having an ill-defined border and a propensity to recur at least as often as conventional ameloblastoma. ${ }^{21}$ 
Following surgical treatment, the patient has remained disease-free for three years and is currently under routine follow-up care. The careful analysis of clinicopathological and radiological findings is recommended to ensure an accurate diagnosis of desmoplastic ameloblastoma.

\section{References}

1. Lam KY, Chan AC, Wu PC, Chau KY, Tideman H, Wei W. Desmoplastic variant of ameloblastoma in Chinese patients. Br J Oral Maxillofac Surg 1998; 36: 129-34.

2. Kawai T, Kishino M, Hiranuma H, Sasai T, Ishida T. A unique case of desmoplastic ameloblastoma of the mandible: report of a case and brief review of the English language literature. Oral Surg Oral Med Oral Pathol Oral Radiol Endod 1999; 87: 258-63.

3. Louis PJ, Fugler RC, August M. Mixed radiolucent/radiopaque lesion of the maxilla. J Oral Maxillofac Surg 2000; 58: 86-90.

4. Manuel S, Simon D, Rajendran R, Naik BR. Desmoplastic ameloblastoma: a case report. J Oral Maxillofac Surg 2002; 60: 1186-8.

5. Pillai RS, Ongole R, Ahsan A, Radhakrishnan RA, Pai KM. Recurrent desmoplastic ameloblastoma of the maxilla: a case report. J Can Dent Assoc 2004; 70: 100-4.

6. Waldron CA, El-Mofty SK. A histopathologic study of 116 ameloblastomas with special reference to the desmoplastic variant. Oral Surg Oral Med Oral Pathol 1987; 63: 441-51.

7. Kaffe I, Buchner A, Taicher S. Radiologic features of desmoplastic variant of ameloblastoma. Oral Surg Oral Med Oral Pathol 1993; 76: 525-9.

8. Ng KH, Siar CH. Desmoplastic variant of ameloblastoma in Malaysians. Br J Oral Maxillofac Surg 1993; 31: 299-303.

9. Wakoh M, Harada T, Inoue T. Follicular/desmoplastic hybrid ameloblastoma with radiographic features of concomitant fibro-osseous and solitary cystic lesions. Oral Surg Oral Med Oral Pathol Oral Radiol Endod 2002; 94: 774-80.

10. Black CC. Addante RR, Mohila CA. Intraosseous ameloblastoma. Oral Surg Oral Med Oral Pathol Oral Radiol Endod
2010; 110: 585-92.

11. Eversole LR, Leider AS, Hansen LS. Ameloblastomas with pronounced desmoplasia. J Oral Maxillofac Surg 1984; 42: 735-40.

12. Effiom OA, Odukoya O. Desmoplastic ameloblastoma: analysis of 17 Nigerian cases. Oral Surg Oral Med Oral Pathol Oral Radiol Endod 2011; 111: e27-31.

13. Reichart PA, Philipsen HP, Sonner S. Ameloblastoma: biological profile of 3677 cases. Eur J Cancer B Oral Oncol 1995; 31B: 86-99.

14. MacDonald-Jankowski DS, Yeung R, Lee KM, Li TK. Ameloblastoma in the Hong Kong Chinese. Part 2: systematic review and radiological presentation. Dentomaxillofac Radiol 2004; 33: 141-51.

15. Poon CS, Wu PC, So MK. Ameloblastoma in Hong Kong Chinese. Hong Kong Med J 1996; 2: 172-6.

16. Li B, Long X, Wang S, Cheng Y, Chen X. Clinical and radiologic features of desmoplastic ameloblastoma. J Oral Maxillofac Surg 2011; 69: 2173-85.

17. Lo Muzio L, Nocini P, Favia G, Procaccini M, Mignogna MD. Odontogenic myxoma of the jaws: a clinical, radiologic, immunohistochemical, and ultrastructural study. Oral Surg Oral Med Oral Pathol Oral Radiol Endod 1996; 82: 426-33.

18. Takata T, Miyauchi M, Ito H, Ogawa I, Kudo Y, Zhao M, et al. Clinical and histopathological analyses of desmoplastic ameloblastoma. Pathol Res Pract 1999; 195: 669-75.

19. Thompson IO, van Rensburg LJ, Phillips VM. Desmoplastic ameloblastoma: correlative histopathology, radiology and CTMR imaging. J Oral Pathol Med 1996; 25: 405-10.

20. Iida S, Kogo M, Kishino M, Matsuya T. Desmoplastic ameloblastoma with large cystic change in the maxillary sinus: report of a case. J Oral Maxillofac Surg 2002; 60: 1195-8.

21. Takata T, Miyauchi M, Ogawa I, Zhao M, Kudo Y, Sato S, et al. So-called 'hybrid' lesion of desmoplastic and conventional ameloblastoma: report of a case and review of the literature. Pathol Int 1999; 49: 1014-8.

22. Philipsen HP, Reichart PA, Takata T. Desmoplastic ameloblastoma (including "hybrid" lesion of ameloblastoma). Biological profile based on 100 cases from the literature and own files. Oral Oncol 2001; 37: 455-60. 